\title{
Comparative Analysis and Modern Applications of PoW, PoS, PPoS Blockchain Consensus Mechanisms and New Distributed Ledger Technologies
}

\author{
Caglar Arslan*, Selen Sipahioğlu, Emre Şafak, Mesut Gözütok, Tacettin Köprülü \\ HAVELSAN Inc., Ankara, 06510, Turkey
}

\begin{tabular}{l} 
A R T I C L E I N F O \\
\hline Article history: \\
Received: 09 July, 2021 \\
Accepted: 01 October, 2021 \\
Online: 14 October, 2021 \\
\hline Keywords: \\
Blockchain \\
Distributed Ledger Technology \\
Crypto Finance \\
Electronic Voting \\
Supply Chain \\
Digital Notary \\
Insurance \\
Records Management \\
Distributed File Systems \\
Artificial Intelligence \\
Federated Learning
\end{tabular}

\section{Introduction}

Blockchain is a distributed database technology in which a database is stored and maintained without the use of a central server across an entire network or among similar parties. No one on the network, inside or outside, has the right to modify or remove recorded data. Transparency is also established by disseminating the data to all chain participants by validating the transmission data via a consensus algorithm [1].

A consensus algorithm is a fault-tolerant mechanism used in computer and blockchain systems to achieve the required

${ }^{*}$ Corresponding Author: Caglar Arslan, HAVELSAN HQ Mustafa Kemal Mah. 2120. Cad. No: 3906510 Cankaya Ankara Turkey, +903122195787, Email: caglara@havelsan.com.tr

www.astesj.com

https://dx.doi.org/10.25046/aj060531 agreement among distributed processes or multi-agent systems on a single data value or a single network state.

A Blockchain infrastructure, as a type of distributed system composed of multiple entities, requires a variant of consensus algorithm in order to operate. The characteristics of any kind of Blockchain are determined by its internal consensus architecture.

A Blockchain, which is defined by a consensus mechanism, enables us to have an immutable database of transaction record among the participating peers. A sample transaction flow is summarized in 'Figure 1' below in order to gain a better understanding of the underlying working process of Blockchain.

\begin{abstract}
Central authorities have registered economic transactions based on trust since the dawn of time. With the advent of paper, methods for documenting transactions and records became technology has advanced, but the maintenance of records has remained under the jurisdiction of the authorities. The use of distributed ledger systems allows data to be network in which non-centralized data is shared by participants. The Bitcoin payment system The aim of Bitcoin is to make the current financial systems liberal from the influence of underpins it has begun to garner interest. While blockchain technology was initially associated with the financial sector due to Bitcoin, research into its use in other sectors such and artificial intelligence has begun. Several Blockchain infrastructures have been built to allow the use of blockchain technology in a variety of industries. However, it has been speed and scalability. As a result, new distributed ledger technologies with increased speed and scalability have been established. Hashgraph, Tangle, Tempo, Holochain are examples of newly developed distributed ledger technologies. Different influential features distinguish new generation distributed ledger technologies from the conventional Blockchain methods, and new generation Distributed Ledger Technologies are compared and possible future applications are outlined.
\end{abstract}




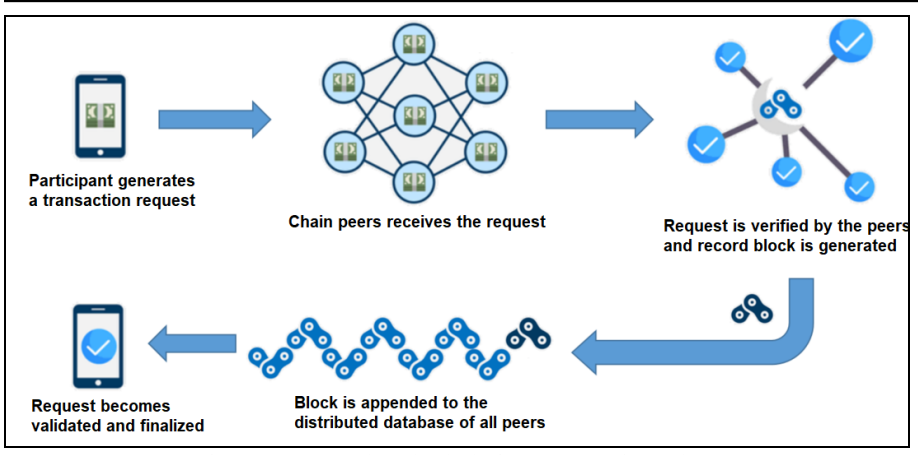

Figure 1: Sample Blockchain Transaction Flow

In summary, the information registry that exists within a group of nodes is made up of multiple blocks of data in which the data is stored and then consolidated within the network after a predefined validation process which is called the consensus.

The necessity of having an immutable distributed record database/table for a digital currency exchange system and applicability of Blockchain to this necessity gave rise to the first useful Blockchain application, which is widely known today as Bitcoin [2]. Since then the blockchain has been synonymous with financial transactions since the rise of Bitcoin, research is currently underway to see whether it can be applied to a variety of areas such as supply chain, records management, energy market, and artificial intelligence.

A variety of consensus algorithms as well as alternative distributed ledger technologies (DLTs), other than the classical Blockchain with the basic Proof-of-Work consensus, have been vastly proposed in recent years each with its own set of performance and security characteristics. It has to be noted that every application's requirements cannot be met by a single consensus algorithm [1]. Hence, it is critical to compare the consistent consensus algorithms as well as other distributed ledger technologies (DLTs) on a technical level in order to identify their strengths, weaknesses, and potential future applications.

Blockchain variants, which are defined by their accompanying consensus algorithms, can have problems regarding throughput, scalability, power consumption and storage usage which can handicap the potential usage areas besides digital currency exchanges/cryptocurrencies. As a result, consistent consensus variants and new distributed ledger technologies (DLTs) should be technically investigated in order to achieve more successful engineering designs of distributed, immutable, manageable, and scalable applications and thus allowing the widespread adoption of trustworthy distributed applications and products in everyday life.

In this study, Proof of Work (PoW), Proof of Stake (PoS), Pure Proof of Stake (PPoS) and new Distributed Ledger Technologies which are named as Hashgraph, Holochain, Tangle and Tempo are introduced and explained. After the introduction and explanations of the mentioned mechanisms, a comparison is made under the performance criteria of Throughput (transactions per second), Scalability (number of nodes), Energy Consumption (J per transaction) and Storage. After this comparison, possible modern applications that can be developed with Blockchain and DLTs are explained and then application-specific minimum performance requirements are given in order to outline the achievability of the applications. Afterwards, suitability of the explained consensus mechanisms and DLTs to each application are explained.

\section{Blockchain and Consistent Consensus Mechanisms}

Blockchain is a decentralized distributed database technology that acts as a trust layer for the data exchange amongst participating peers.

The following key features define any type of Blockchain: Immutability, transparency, privacy, durability, decentralism, and security.

- Immutability: No participant can change the data stored in the blockchain.

- Transparency: Transparency is provided to all participants in the Blockchain by associating the transaction data with the concerning participants. All transactions are traceable.

- Privacy: The use of private and public keys is a crucial aspect of blockchain privacy. Asymmetric cryptography is used in blockchain systems to secure transactions between users.

- Durability: A Blockchain can operate indefinitely since a copy of the data is distributed across the participating nodes.

- Decentralism: The transfer of control and decision-making from a centralized entity (individual, organization, or group thereof) to a distributed network is referred to as decentralization in blockchain.

- Security: The structure of data created by blockchain technology has built-in security properties. Trust in transactions is ensured by the use of cryptographic principles, decentralization, and consensus. Each new block in a cryptographic chain connects to all the blocks before it in such a way that tampering is nearly impossible. A consensus mechanism validates and agrees on all transactions within the blocks, ensuring that each transaction is true and correct.

It has to be noted that throughout this study, Blockchain is referred as the two most popular consensus mechanisms namely the Proof of Work (PoW) and Proof of Stake (PoS) and a new consensus algorithm that is consistent with the key Blockchain features (i.e. decentralized, secure and scalable) and named as Pure Proof of Stake (PPoS).

\subsection{Proof of Work (PoW) Consensus}

The PoW consensus mechanism has been popularized by Bitcoin as one of the most widely used methods in blockchain (BTC). Miners and the electricity needed to perform the calculations that verify BTC transactions are the distinguishing features of PoW systems. Miners use computer hardware to run network nodes that use computational power to solve mathematical puzzles known as proofs of work. The miner who solves the puzzle first confirms the most recent block of transactions on the blockchain.

The successful miner then broadcasts the new block to all other nodes, who confirm its accuracy and add it to their copy of the blockchain, creating a verifiable record of data for the entire network. Consensus is represented by the verification process. A new block can only be added to the network after this data has been confirmed. For being the first to validate a new block of data and 
add it to the PoW blockchain, miners receive newly minted cryptocurrency, the block reward (in the case of Bitcoin, they receive $\mathrm{BTC})$.

Proof of Work blockchains aim to generate blocks at regular intervals - for example, Bitcoin generates one block every ten minutes. PoW networks are limited in speed and scale because of the energy-intensive proofing process. Furthermore, PoW networks are engineered to be more or less challenging depending on the network's computing capability.

Despite their speed and scalability restrictions, PoW blockchains have typically given better security while maintaining significant decentralization. Because PoW systems are distributed, it is extremely difficult for a malicious actor to take control of the blockchain by controlling the majority of computing power on the network. Typically, the hardware, power, and computing expenses are prohibitively expensive [3].

However, the same characteristics that make PoW blockchains secure also make it difficult to participate in the network as a node. Mining on many major networks has been monopolized by largescale mining operators who have amassed influence in network governance. Since operating a mining rig and paying for the associated hardware and electricity costs is too expensive for the average user, large-scale mining operators have collected major influence in the network governance.

\subsection{Proof of Stake (PoS) Consensus}

PoS is the second most often used consensus method, and it addresses many of the issues that plague PoW blockchains, such as slowness, scalability, inefficient energy usage, and expensive requirements for entering the network.

PoS blockchains use validators instead of miners to validate transactions. Validators are network node operators who validate data in the same way that PoW systems do, but there is no energyintensive computational process required to earn the right to validate. Instead of doing proofs of work, validators "stake" part of the blockchain's native currency in order to be considered as a validator node. The prospective validator mainly uses blockchainborn crypto tokens to provide collateral. The system randomly selects a validator to confirm data when validating the data held in a transaction block on a PoS blockchain. Certain factors, such as the number of tokens staked, can increase the likelihood of a validator being chosen. The validator is rewarded with network transaction fees when a block is confirmed, and then the process starts over [3].

Proof-of-Stake blockchains keep the network secure and validators honest by demanding validators to stake their tokens. If validators are acting deliberately or incompetently, their involvement and access to the network is lost by a 'slashing' procedure. This stimulus structure makes it possible for validators to earn through legal operation rather than by breaching regulations.

Accessing the PoS blockchains is easier as the validators on the PoS blockchains do not need to invest in high electricity costs and expensive hardware. PoS blockchains are arguably better for the environment than PoW networks in terms of sustainability because they consume significantly less electricity [4].

\subsection{Pure Proof of Stake $(P P o S)$ Consensus}

PPoS is a new fully decentralized and promising consensus mechanism. The amount of influence each user has over the selection of a new block is proportional to their stake (number of tokens) in the system. Users are chosen at random and in secret to propose and vote on block suggestions. Every online user has the opportunity to be chosen to propose and vote. The probability of a user being picked, as well as the weight of its proposals and votes, is proportionate to its stake.

This means that only two blocks of PPoS cannot be sent to the chain simultaneously since the required threshold of committee votes can be reached by a single block. Up to 1 block in a given round is confirmed and written on the chain. When a block is created, users may immediately depend on the transactions it includes. Thus users can rely on that the block, since it will be part of the chain forever.

PPoS does not place a small group of users responsible for generating blocks, and users do not have to transfer their voting power to a limited number of users. All users are allowed to propose and vote on blocks with a probability that is directly in proportion to their stake.

PPoS doesn't force a user to put a part of their stake aside for a consensus process, and a user can not restrict his ability to spend their stake by participating in a consensus protocol.

In a completely decentralized fashion, PPoS guarantees full participation, protection and speed [4].

\section{Distributed Ledger Technologies}

The authors first mentioned distributed ledger technology in their 1991 article "How to time-stamp a digital text" [5]. Distributed ledger technology (DLT) is a decentralized framework in which data is shared among network participants. Despite the fact that the approaches to distributed ledger technology are technically distinct, they all have three fundamental components in common.

Every distributed ledger technology should support peer-topeer transactions, have participants who store the network, and use a consensus mechanism to manage it.

The ability of two or more parties to operate without the use of a central authority is known as a peer-to-peer network. Participants in a distributed ledger are independent computation nodes that record, share, and synchronize transactions.

DLTs are managed without the central authority being required [6]. With the independent conduct of transactions, the efficiency can be improved by reducing errors.

To address the speed and scalability issues in Blockchain technology, new distributed ledger technologies have emerged, such as the Tangle, Hashgraph, Tempo, and Holochain. This newly developed distributed ledger infrastructures aim to improve blockchain technology's limited features.

It has to be also noted that every Blockchain is a type of DLT but not every DLT is a Blockchain [7]. 


\subsection{Hedera Hashgraph}

Swirlds developed Hashgraph, a proprietary distributed ledger technology. The consensus algorithm and the Directed Acyclic Graph (DAG) data structure distinguish it from Blockchain technology. Hashgraph does not use conventional Blockchain consensus. Instead of conventional consensus mechanisms, it employs the random gossip protocol and the virtual voting consensus algorithm.

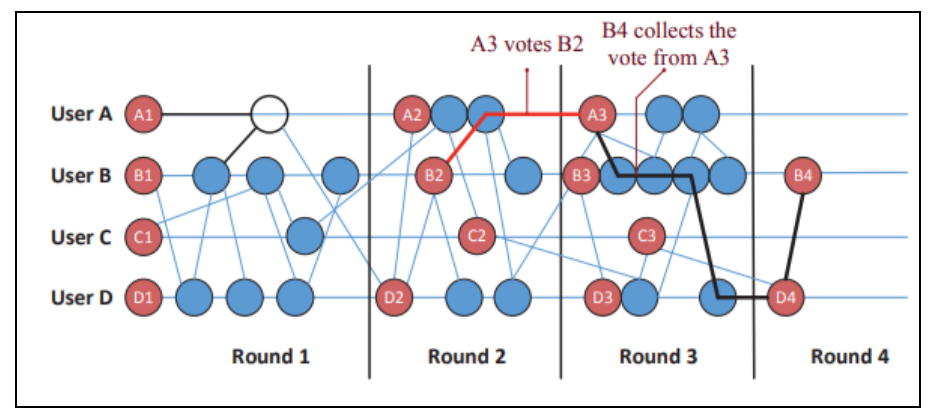

Figure 2: An example of Hashgraph’s operation. Image courtesy of [8]

Any transaction that occurs in the Hashgraph network is referred to as an event. Each event includes a timestamp, a hash of the previous two events, transactions, and electronic signature information. The random gossip protocol distributes the time stamp of the verification process to neighboring nodes at random until the data is applied to the ledger [9]. Refer to the Figure 2 as an example of the Hashgraph algorithm.

In order to validate the transaction, this information must be transmitted to the majority of network participants. Transaction timestamps are documented in the case of a separate hashgraph ledger maintained by the participants. Transactions that are agreed by two-thirds of the network through virtual voting are considered legitimate and are registered by each node. Virtual voting can require several rounds in the absence of a majority of participants,

\subsection{Tangle}

A directed acyclic graph (DAG) is a specialized subject in mathematics, particularly graph theory and computer science. A DAG is made up of vertices and edges (also known as arcs), with each edge pointing from one vertex to another in such a way that following those directions will never result in a closed loop. This mathematical model is being considered as an alternative approach for developing alternative Blockchain structures for applications consisting of many devices [10].

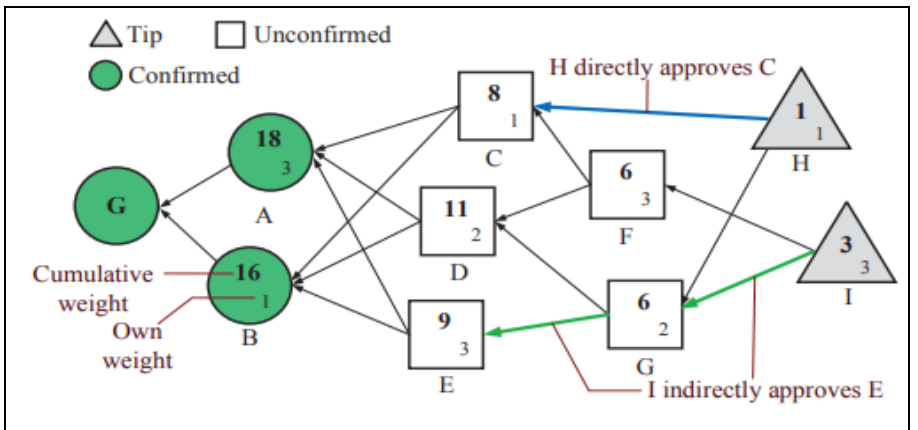

Figure 3: An example of Tangle’s DAG based operation. Image courtesy of [8]
Tangle is a new type of DLT which is considered as one of the major DLT that vastly uses the DAG model. Refer to the Figure 3 for visualizing the Tangle's operation.

Since the current mainstream Blockchain infrastructures (i.e. PoW) are unable to meet the efficiency and scalability requirements of IoT systems that require a Trust Layer, Tangle DLT is becoming increasingly important in establishing trust and security for the Internet of Things (IoT) applications.

There is no transaction fee in Tangle since there are no blocks or miners to add data to the network. Transactions are not kept in a sequential order. Transactions are not recorded in a linear fashion. Each transaction in the consensus protocol verifies the previous two transactions and records the transaction data. In terms of security, it is less secure than Blockchain technology especially in the early stages of the network. When the transaction volume is low, network coordinators are used for protecting the network.

The use of network coordinators may result in the network not being fully distributed. The goal is to improve the scalability, speed, and efficiency of the Tangle protocol. The speed of the network increases in direct proportion to its size, especially as the network grows.

Tangle, which is used in IOTA, is one of the most important applications that use the DAG model [11].

It is appropriate to use this advanced DAG based DLT particularly in IoT applications or any other applications that require providing speed and scalability to a network composed of large number of devices and transactions.

\subsection{Holochain}

Holochain is an open-source distributed ledger technology. Unlike Blockchains, Holochain relies on individual chains rather than a single chain. While transactions in Blockchain technology are made over a single chain for all network participants, participants in Holochain store data in their local chains as part of the public network, Instead of recording all of the data on the network, participants only record their own transactions. This situation should be considered as the main reason why this DLT requires low amount of storage on participating nodes. The Distributed Hash Table (DHT) [12] ensures the correctness of local chains. DHT stores the chain data's DNA (hash) information and ensures that the chain's invariance is maintained. As indicated in Figure 4, DHT is stored in parts of the mesh topology [13]. While some users are offline, the network can continue to function. The disadvantage of this protocol is that information can be lost if a registered peer's device completely malfunctions. A backup procedure is additionally required for all peers in order to prevent data losses.

Distributed applications (dApps) built on the Holochain,which are named as Holochain Apps (hApps), can run quickly, and the network's scalability is limitless. Millions of transactions can be processed per second with Holochain. Holochain aspires to transform the current internet's center-based services.

\subsection{Tempo Radix}

Sharding method is used by Tempo distributed ledger technology in order to distribute data amongst many nodes in the 
network. Hence, this situation eases the amount of storage required on the peers.

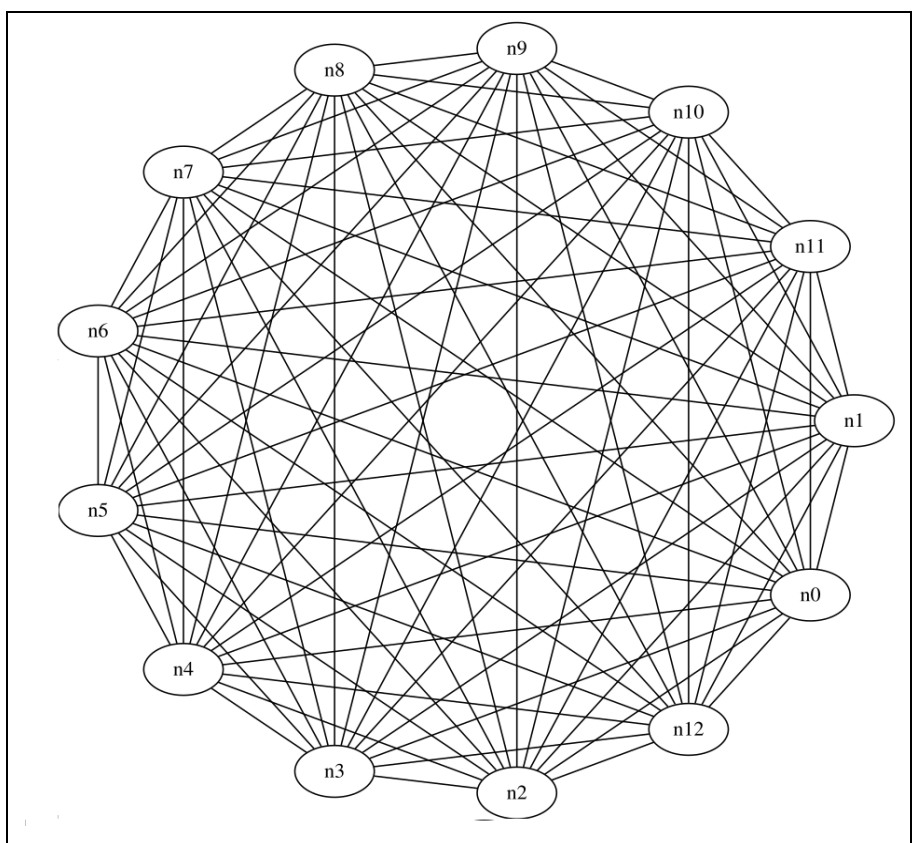

Figure 4: A representation of Holochain's DHT topology. Image courtesy of [13]

Tempo relies on Lamport's Logical Clocks, see Figure 5 for a sample representation of this concept, to come to an agreement on the distributed ledger. Any time a transaction is recorded, the logical hour number is also recorded. Each node increments its logical counter for every transaction it witnesses. Due to the unreliability of using the physical clock in distributed systems, the logical clock system is used. Tempo logical hours are smart counters that count upwards in increments of one when a participant sees a new and verified transaction in the ledger,

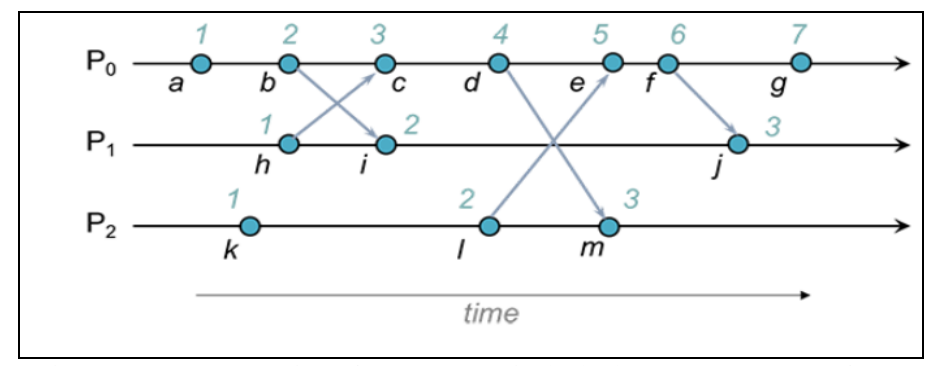

Figure 5: A representation of Lamport Logical Clocks. Image courtesy of [14]

Tempo participants use the Gossip protocol in order to synchronize the timeliness of the information in their tracks [15].

Participants in a blockchain network have access to the entire ledger, but the Tempo infrastructure uses shredding technology to limit participants' access to a subset of the ledger. Some sections of the participants' data are also being recorded by neighboring nodes as a data-loss prevention mechanism.

\section{Comparison of PoW, PoS, PPoS Blockchain Consensus Mechanisms and Distributed Ledger Technologies}

\subsection{Blockchain versus Hedara Hashgraph}

In terms of speed and scalability, Hedera Hashgraph can outperform the Blockchain technology. The transaction volume is high due to the Hedera Hashgraph's random gossip protocol, which allows transactions to be released quickly to the network. When a node conducts a transaction, it transfers transaction information to a randomly chosen neighbor node [9]. The node that discovers the transaction will also pass this information on to a random neighbor node. This procedure is repeated until the entire network is aware of the information. As a result, the network is immediately alerted when a new transaction occurs.

Hashgraph DLT uses virtual voting to validate each transaction, rather than the costly mining process in some Blockchain infrastructures. If two-thirds of the entire network accepts the transaction, the transaction is valid. Virtual voting can take place several times if the majority is not reached. In Hedera Hashgraph distributed ledger technology, a maximum of 250,000 transactions per second can be performed [16].

Hedera Hashgraph is more scalable than Blockchain. The Hedera Hashgraph network can potentially have an infinite number of participants.

\subsection{Blockchain versus Tangle}

Tangle distributed ledger technology has increased scalability and quantum durability compared to Blockchain technology, while reducing transaction fees in open blockchains.

To confirm a transaction in Tangle, each node must verify at least two transactions from previous transactions. The participant's transaction becomes more valid as it validates more transactions. It is the most resistant distributed ledger technology even it is also considered as quantum-safe.

Tangle arises amongst the other DLT alternatives with its lowest transaction fee. When compared with Blockchain, the security of Tangle is weaker when the network size is smaller.

The number of transactions per second in Tangle DLT his estimated between 500 and 800 [17]. Due to the nature of Tangle's operation, it is assumed to allow an unlimited number of participants.

Tangle infrastructure can be preferred in applications that require speed and scalability such as instant mobile voting applications. Also, it is allegated that Tangle can also have a good impact on the Energy Trade industry [8].

\subsection{Blockchain versus Holochain}

In comparison to Blockchain, resources are used efficiently and scalability is increased with Holochain. Data on the network is kept in separate chains rather than a common ledger in Holochain technology .

As part of the public network, each participant keeps its own local records. Each participant keeps a separate ledger. Distributed Hash Table (DHT) ensures the accuracy and immutability of local ledgers. Holochain DLT allows a truly distributed architecture in which everyone maintains their own ledgers.

As opposed to Blockchain technology, energy efficiency is ensured by avoiding costly mining operations via the Holochain consensus mechanism. 
Since there is a central point through which all transactions must pass, Holochain does not set a TPS (transactions per second) value like other blockchain-based or blockchain-derived projects [18]. Holochain, on the other hand, is a generalized distributed computing protocol. Due to the underlying mechanisms, throughput and scalability are assumed as theoretically infinite.

\subsection{Blockchain versus Tempo Radix}

In terms of speed, scalability, and resource efficiency, Tempo distributed ledger technology can outperform Blockchain. As opposed to Blockchain's single chain, Tempo distributes the subportions of the entire ledger onto the network participants' storage. In other words, Tempo participants store the puzzle pieces by viewing the entire ledger as a puzzle [15].

Each node keeps a piece of its neighbor node in the fragmentation process in order to prevent data loss. Tempo distributed ledger technology's shredding process has advantages in terms of speed and resource efficiency. In Blockchain, a lot more resources are used since the entire block data / ledger is kept on all participants. Additionally, distributed ledger synchronization is slower and less scalable than Tempo distributed ledger technology.

Tempo distributed ledger technology has been tested to handle 1,400,000 transactions per second [19]. The Tempo network has a significant advantage over Blockchain technology because the maximum number of participants is theoretically unlimited.

The Gossip protocol is used in Tempo distributed ledger technology ensures that nodes have up-to-date information about the distributed ledger. The Gossip protocol allows the nodes in a network to propagate new configurations to the rest of the network. This protocol allows network nodes to communicate with one another and exchange information about their components.

\subsection{Comparison of Blockchain (PoW, PoS, PPoS) and DLTs}

In a typical setting, performance and evaluation metrics for developing a distributed application depends on four key parameters, Throughput (total number of transactions per second on the distributed network), Scalability (Supported number of nodes in the distributed network), Energy consumption per transaction and the level of storage requirement/usage.

'Table 1' summarizes the key performance and evaluation metrics of the consensus mechanisms and DLTs which are outlined in this study.

Throughput, scalability values and the storage usage levels are gathered from the internally conducted research and from the following references: [16], [17], [20]-[22].

For the energy consumption of Blockchain consensus protocols, in a network of 10000 nodes, which is approximately the number of active full nodes on PoS, note that this amounts to approximately $1000 \mathrm{~J}$ per transaction for PoS, it is many orders of magnitude less than for the current PoW blockchains which is about 1 billion $\mathrm{J}$ per transaction [23]. It has to be also noted that PPoS uses $30 \mathrm{~J}$ per transaction [24].

As for the DLT segment, Tangle uses 3.6 J per transaction [25]. It is claimed that Hashgraph uses $3600 \mathrm{~J}$ per transaction [26]. Holochain's energy consumption is estimated as $325 \mathrm{~J}$ per www.astesj.com transaction [27]. Energy consumption of Tempo is not available in the literature, however it is considered very low since no mining is involved [28].

Table 1: Key performance comparison and evaluation metrics of Blockchain (PoW, PoS, PPoS) and DLTs

\begin{tabular}{|c|c|c|c|c|}
\hline & $\begin{array}{l}\text { Throughput } \\
\text { (transactions } \\
\text { per second) }\end{array}$ & $\begin{array}{l}\begin{array}{l}\text { Scalability } \\
\text { (number of } \\
\text { nodes) }\end{array} \\
\text { no }\end{array}$ & $\begin{array}{l}\text { Energy } \\
\text { Consumption } \\
\text { (J per } \\
\text { transaction) }\end{array}$ & Usage of Storage \\
\hline $\begin{array}{l}\text { Blockchain } \\
\text { - PoW }\end{array}$ & $\sim 7$ tps & $\sim 10.000$ nodes & $\begin{array}{l}\sim 1 \text { billion } \\
\mathrm{J} / \text { transaction }\end{array}$ & High \\
\hline $\begin{array}{l}\text { Blockchain } \\
\text { - PoS }\end{array}$ & $\sim 25$ tps & $\sim 10.000$ nodes & $\begin{array}{l}1000 \\
\mathrm{~J} / \text { transaction }\end{array}$ & High \\
\hline $\begin{array}{l}\text { Blockchain } \\
\text { - PPoS }\end{array}$ & $\sim 10000$ tps & 500.000 nodes & $\begin{array}{l}\sim 30 \\
\mathrm{~J} / \text { transaction }\end{array}$ & High \\
\hline $\begin{array}{l}\text { DLT } \\
\text { Hashgraph }\end{array}$ & $\begin{array}{l}\sim 250.000 \text { tps } \\
\text { (Theoretically) } \\
\sim 10.000 \text { tps } \\
\text { (recent practical } \\
\text { result) }\end{array}$ & $\begin{array}{l}\text { Theoretically } \\
\text { Unlimited }\end{array}$ & $\begin{array}{l}3600 \\
\mathrm{~J} / \text { transaction }\end{array}$ & Moderate \\
\hline $\begin{array}{ll}\text { DLT } & - \\
\text { Tangle } & \end{array}$ & $\sim 500$ tps & $\begin{array}{l}\text { Theoretically } \\
\text { Unlimited }\end{array}$ & $3.6 \mathrm{~J} /$ transaction & Moderate \\
\hline $\begin{array}{l}\text { DLT } \\
\text { Holochain }\end{array}$ & $\begin{array}{l}\text { Theoretically } \\
\text { Unlimited }\end{array}$ & $\begin{array}{l}\text { Theoretically } \\
\text { Unlimited }\end{array}$ & $325 \mathrm{~J} /$ transaction & Low \\
\hline $\begin{array}{ll}\text { DLT } & - \\
\text { Tempo }\end{array}$ & $\sim 1.400 .000$ tps & $\begin{array}{l}\text { Theoretically } \\
\text { Unlimited }\end{array}$ & $\begin{array}{l}\text { N/A } \\
\text { (Considered } \\
\text { very low) }\end{array}$ & Low \\
\hline
\end{tabular}

\section{Modern Applications of Blockchains and Distributed Ledgers}

In empirical settings, blockchain and distributed ledger technologies can be used in a variety of ways. Like all technologies, a Blockchain application must be viewed as a sociotechnical system. A sociotechnical system consists of a technological artifact and the social environment in which it is used, as well as the interactions between the two. When viewed as a sociotechnical system, the core characteristics of Blockchain and DLTs can be applied to a variety of situations, resulting in a wide range of use cases.

\subsection{Cryptocurrency}

A cryptocurrency is a digital or virtual currency that is mostly based on consistent Blockchain consensus mechanisms and protected by cryptography, making counterfeiting and doublespending nearly impossible. Cryptocurrencies are distinguished by the fact that they are not issued by any central authority thus allowing them to be theoretically immune to government intervention or manipulation.

Cryptocurrencies have the potential to make it easier to transfer funds between two parties without the use of a trusted third party such as a bank or credit card company. In addition, users can avoid the high fees charged by banks and financial institutions for wire transfers by preferring cryptocurrency based fund transfers with minimal processing fees [29].

Major disadvantageous situation of Cryptocurrencies is that the semi-anonymous nature of Cryptocurrency transactions makes them ideal for a variety of illegal activities, including money laundering and tax evasion. However, Bitcoin is a poor 
choice for conducting illegal operations because forensic analysis of the Bitcoin has aided authorities in detecting criminal activity. It has to be noted that more privacy-oriented coins like Dash, Monero, and ZCash, on the other hand, are far more difficult to track than the Bitcoin [30].

\subsection{Decentralized Finance (DeFi)}

Decentralized Finance, also known as DeFi, is a movement that allows users to access financial services such as borrowing, lending, and trading without relying on centralized entities. These financial services are delivered through Decentralized Applications (Dapps), the vast majority of which are built on the PoS powered Ethereum platform [31].

DeFi is a collection of products and services that act as a replacement for institutions such as banking, insurance, bonds, and money markets, rather than a single product or company. DeFi Dapps allow users to combine their services in order to expand their options. DeFi Dapps typically require collateral to be locked into smart contracts in order to function. The Total Value Locked is a term used to describe the total collateral locked in DeFi Dapps [32].

By eliminating the need for any middlemen, DeFi Dapps have the potential to revolutionize traditional financial services. It should be noted, however, that DeFi in its current state is still very new and experimental, with many projects being improved on a daily basis. DeFi may evolve further over time and become completely unrecognizable from what it is now.

\subsection{Non-Fungible Tokens (NFT)}

NFTs (non-fungible tokens) are a new type of blockchainbased token that is one-of-a-kind and indivisible. They first appeared in late 2017. NFTs are digital tokens that can be used to show who owns one-of-a-kind items. Unlike PoW powered Bitcoin, where each coin is identical, NFTs are unique, with their own set of features. Tokenized items include art, valuables, and even real estate that no one can alter the ownership record. NFTs can only have one official owner at a time and are mainly secured by the PoS based Blockchain infrastructures [33].

\subsection{Digital Identity}

Any document that can be used to prove an individual's identity is known as an identity document. We frequently share our identity information to authenticate in our daily lives. Personal data is violated when identity information is stored, processed, and shared against the person's will. There is no universally accepted identity in the Internet.

A digital identity system can be used for information confirmation as well as authentication. Users will have complete control over their digital ID and personal information. Verification can be handled with or without sharing entire personal information in the digital identity system.

Blockchain has progressed significantly since its inception as a distributed ledger system for tracking bitcoin ownership. This technology has the potential to replace traditional identity management systems with a highly trusted mechanism. Users will be able to have more control over their own identities owing to the infrastructure provided by the Blockchains [34] and DLTs.
Organizations can only use the data with the consent of customers, and no central entity will be able to compromise a customer's identity. Self-sovereign identity, which is inherently unalterable and more secure than traditional identity systems, has been made possible by Blockchain [35]. This has the potential to completely transform how we connect to various online services using our identities. Individuals would verify their identity using their self-sovereign ID, eliminating the need for passwords.

As with any life-changing innovation, there has been a long period of evolution, with experts exchanging ideas and little agreement on what self-sovereign ID means. It is based on the idea that an individual must have control over how his identity is managed. With user consent, the ID cannot be tied to a single system and must be interoperable across multiple platforms. Experts considered combining various identifying data, such as demographic and employment-related information, as well as individual information revealed by others.

In the Blockchain domain, digital identities and user information will be stored in a digital wallet. There will be no need to carry or store many cards or documents. In a Blockchain based digital identity system, documents such as our citizenship card, bank cards, diplomas, certificates, driver's license, and passport are being planned to be stored in self-sovereign id oriented digital wallets.

\subsection{Supply Chain Management}

One of the most important areas in which blockchain projects are concentrated is considered as supply chain management. A trust issue arises because of the large number of parties involved in supply chain processes. Supply chain transaction information is being planned to be securely stored by Blockchain [36]. The current method of running these processes on paper makes it difficult to manage and unreliable. By using Blockchain, it is possible to conduct transactions securely on digital media and to view all stages of the processes transparently with confidence. Circulation of counterfeit products can also be prevented from the market using this approach. Preventing the sale of smuggled goods will protect citizens' rights and countries from incurring tax losses.

Consumers may not fully confirm whether the procured products were genuine or not in a traditional supply chain process. Consumers can reliably view the production and distribution history of products by using Blockchain enhanced Supply Chain Management Systems. With this understanding in mind, countries may want to look at the manufacturing, distribution, and shipping history of all imported goods in the future.

\subsection{Digital Notary Services}

A notary, also known as a public notary, is a state-appointed official who has the authority to certify a specific action or deed. Contracts, documents, affidavits useful in other jurisdictions, and deeds are all included. In order to perform the role of notary, an official must be objective when making various declarations. A notary's duties include everything from signing a property sale record to administering oaths and keeping accurate records of other important deeds and documents. The officer can use an 
electronic or digital notary to perform notarizing functions digitally.

The authorized seal will be applied to the certified document by the electronic notary. This activity requires cryptography and a secure public key in order to manage, develop, store, and distribute the digital certificate since a digital notary must keep an up-to-date electronic record of all completed notary tasks. Remote implementation of a significant digital solution for public notary activities is also possible. This notarization process is expected to be aided by Blockchain and DLT technology. As a prospecting result, users of this service can trust the integrity of data on the Blockchain or DLT.

Nonrepudiation, tamper-resistance features and the wellknown characteristics of distributed ledgers make it an excellent tool for enhancing notary capabilities [37].

\subsection{Land Registry and Property Ownership Management}

Land registration entails gathering information such as the ownership and property size information of a land piece. The entire process of land registry maintenance is extremely timeconsuming because it demands the safekeeping of massive volumes of written registers. The current method is insecure since the majority of this procedure is opaque and continuous sales of a property must be accurately recorded. There have been several proposals to automate land register data recordings by eliminating the need for handwritten records. This is initially accomplished by storing the data in massive databases. However, in terms of data security, such a strategy is ineffective because the data contents are easily accessible, and data tampering can occur in poorly maintained databases.

A distributed ledger based solution for property registration could significantly improve efficiency and possibly eliminate fraud [38]. Contracts and ownership data can be stored in a distributed manner too. Blockchain and DLT implementations can make it easier to track data transactions because it eliminates the need for physical involvement thus enhancing the overall system security for users. Developing countries are considering Blockchain and DLTs as a tool for updating their existing land registry systems.

\subsection{Health Records Management}

Preventing illnesses or detecting them early would improve society's living conditions and reduce the workload of hospitals. Citizens' health status should be constantly tracked using preventive health technologies. The volume of health data will grow as a result of constant surveillance of citizens' health status. This data can be immutably stored and exchanged for clinical trials using Blockchain and DLT technology.

Health data is vital information that countries must safeguard. Theft of these details poses a threat to the country's national security. Health information is also essential to the pharmaceutical industry. As a result, health data has become an obvious priority for financial benefit.

Blockchain and DLTs can serve as a link between healthcare researchers and health data records in order to facilitate data exchange. Blockchain and DLTs can provide secure storage and exchange of health data at any time. Anonymized health data can have economic benefits to both users and organizations [39]. A health consortium with Blockchain infrastructure, comprised of organizations that generate and need data, can be created. As a result, the data can be safely exchanged with the desired entities and organizations for scientific purposes.

\subsection{Insurance}

The insurance industry can use Blockchain and DLT technology in order to improve core internal processes like claimsubmission and processing, as well as fraud detection and prevention. Blockchain can also be used to modify and possibly create new insurance brands, as well as improve existing ones. It has also come to light that Blockchain faces a number of issues, including scalability, security, and privacy, as well as taxation and regulation. The insurance industry has yet to reap the benefits of new generation blockchain architectures and distributed ledger technologies [40].

\subsection{Electronic Voting Systems}

It has long been a challenge to create a secure voting structure that offers the same fairness and privacy of current voting schemes while also providing the transparency and flexibility of electronic systems. To compete with the conventional ballot system, an electronic voting system must meet the same security and privacy criteria.

A Blockchain or DLT based e-voting technology has the ability to reduce election fraud while also increasing voter access [41]. Such an e-voting system could decentralize controls, enabling electors to take on certain duties while maintaining a copy of the electoral register. Since the copies of the ballots are a voting history that cannot be reversed, there can be no null votes [42].

\subsection{Energy Distribution and Trade}

Given the finite nature of resources, countries must increase their production of renewable energy resources. As a result, the number of local producers who use renewable energy is expected to grow in the future. The structure of energy distribution and trade will be altered as the number of local energy producers increases. To encourage local producers, an energy trading platform would be developed, allowing producers to load their excess energy to the grid immediately.

It is necessary to ensure that energy exchange between the local producer and the consumer is secure. Blockchain and Distributed ledgers could be used to create a platform that allows local producers and consumers to transact usage certificates instantly. For such an energy distribution and trade infrastructure, all parties involved in the energy distribution process must form a distributed ledger based consortium. In the energy sector, Blockchain and DLT will bring together market regulators, distributors, consumers, home users, and producers on a single, reliable energy market and sharing platform [43].

This platform will enable real-time allocation of required and preferred types of energy, as well as the approval of energy consumption. This platform would provide incentives to renewable energy producers. Blockchain has the potential to provide an infrastructure for instant energy trade for energy consumers and local conventional or renewable energy producers [44]. Smart meters will also need to be developed in order to measure the produced or purchased energy. 
Active customer engagement and energy trading would require immutable, open, and dependable smart contracts. Such digital energy market systems can ensure that energy is priced immediately and it is delivered on demand, and allows the customer to determine their own energy consumption preferences. It would also make the consumer more efficient in deciding the frequency of energy usage.

It has to be noted that especially for the energy industry, DAG based structures can have a critical role [8].

\subsection{Distributed File Systems}

Distributed file systems (DFS) have gained a lot of interest recently. Instability, auditing, and incentive mechanisms are all weaknesses in traditional Peer-to-Peer (P2P) distributed file systems. Although Blockchain-based DFSs successfully provide enough incentives and security assurances, a number of problems such as scalability and privacy concerns are preventing the next generation of Blockchain DFSs from being developed. As a result, it is anticipated that DLTs have much greater potential than conventional Blockchain in order to establish a globally distributed file system due to their storage-wise and scalability advantages [45].

\subsection{Artificial Intelligence and Federated Learning}

A new machine learning paradigm known as federated learning (FL) has arisen as a result of the increasing computing capabilities at end user devices, as well as the growing privacy concerns over sharing sensitive raw data. FL has the potential to avoid exchanging data directly by training models locally at each client and aggregating learning models at a central server, hence avoiding privacy leaks. The standard FL structure, on the other hand, is strongly reliant on a single central server, which could fail if that server acts maliciously. To address the single point of failure problem, new approaches are being developed that focus on establishing a DLT-assisted decentralized FL framework that can effectively prevent malicious clients from poisoning the learning process while also providing clients with a self-motivated and reliable learning environment.

Distributed Ledger Technologies have the potential to improve transparency and trust in the distributed machine learning process and generating consistent models by permanently logging it into a distributed ledger. Since the model is available to the public and the history is preserved, the blockchain tracks not just the current model but also all of the previous iterations. Since decentralization is inherent in both, federated learning can benefit from distributed ledgers [46].

\section{Application-specific Minimum Performance Requirements for Achievability of the Applications}

Minimum required levels of performance requirements for the above mentioned applications are given in Table 2. This section does not provide the desired ideal state of applications. It has to be noted that; there does not exist any universal solution for all types of applications. Hence, it is necessary to outline the needed basic and minimum performance requirements for having a better decision-making action in order to select an appropriate protocol or conduct a better protocol implementation strategy during the initial phases of a specific distributed project. All of rationals of Table-2 are given in the subsections.
A scoring system of Low, Moderate and High is used in order to outline the minimum requirements. It has to be that ' $\mathrm{DC}$ ' denotes, as a scientific and engineering abbreviation, "Don't Care”.

Table 2: Application-specific Minimum Performance Requirements for the Achievability of the Applications

\begin{tabular}{|l|l|l|l|l|}
\hline & $\begin{array}{l}\text { Throughput } \\
\text { (transactions } \\
\text { per second) }\end{array}$ & $\begin{array}{l}\text { Scalability } \\
\text { (number of } \\
\text { nodes) }\end{array}$ & $\begin{array}{l}\text { Energy } \\
\text { Efficiency } \\
\text { (J p/tx) }\end{array}$ & $\begin{array}{l}\text { Level of } \\
\text { Storage } \\
\text { Requirement }\end{array}$ \\
\hline Crypto Currency & Low & Low & DC & DC \\
\hline DeFi & Low & Moderate & Moderate & DC \\
\hline NFT & Low & Low & DC & DC \\
\hline Digital ID & Moderate & DC & DC & Moderate/High \\
\hline Supply Chain & Moderate & Moderate & DC & High \\
\hline Digital Notary & Low & Low & DC & High \\
\hline Land Registry & Low & Low & DC & Moderate \\
\hline Health Records & Moderate & Low & DC & High \\
\hline Insurance & Moderate & Low & DC & Low \\
\hline $\begin{array}{l}\text { Electronic } \\
\text { Voting }\end{array}$ & High & High & High & Moderate \\
\hline Energy Trade & Moderate & High & High & Moderate \\
\hline DFS & High & Moderate & DC & High \\
\hline AI/FL & Moderate & Moderate & DC & High \\
\hline
\end{tabular}

\subsection{Cryptocurrency - Minimum Performance Requirements}

Cryptocurrency applications can be achieved with low rate of transactions per second and low number of participating nodes. Any cryptocurrency system can be achieved with low levels of transaction rate with a small number of validating or participating nodes. The most basic and stable example of this situation can be given as the PoW based Bitcoin in which the performance metrics are given in Table 1.

\subsection{DeFi - Minimum Performance Requirements}

As in the case of Cryptocurrency, any DeFi project can be achieved with low transaction rate. Major difference is the necessity of having a more energy efficient and moderately scalable infrastructure for the purpose of having a more futureproof DeFi system. The reason is that; there is the possibility of having multiple mobile devices participating in the DeFi domain in the near future.

\subsection{NFT - Minimum Performance Requirements}

NFT portals or NFT systems recently does not have and need many transactions per second and participating nodes, since NFTs are used mainly for the occasional transfer of the ownership of any concept.

\subsection{Digital ID - Minimum Performance Requirements}

The required transactions of Digital ID records will be moderately high enough. In an interconnected e-government environment, the transactions between the official Digital ID issuers, validators and peers will be moderately high enough in a daily setting. This situation will also be true for keeping and updating the Digital ID records of the objects.

Considering the large number of files in a countrywide setting, the storage requirement will be essentially very high for the issuer and verifier sides of a Digital ID system. 
As a special note; if there exits multiple IoT (Internet of Things) devices in an Digital ID system then the level of storage requirement needs to be moderately large enough in order to store the entire history of record in the IoT node's lifecycle.

\subsection{Supply Chain - Minimum Performance Requirements}

In a worldwide supply chain setting, the amount of transactions between suppliers, manufacturers, distributors, retailers and consumers is considered very large. As a result, the required level of storage is needed to be large enough in order to collect and store the entire decades of transaction records.

The required minimum transactions need to be moderately high enough due to the speed requirements in a global environment for a supply chain operation and considering the amount of participating nodes in this setting, the scalability level should also need to be moderate.

\subsection{Digital Notary - Minimum Performance Requirements}

On a daily urban life setting, the necessity and frequency of notary services is considered high. In a Blockchain/DLT based Digital Notary scenario, the notary branches in a city can be considered as validating nodes. When compared with any large scale application, the number of notary branches are not large enough and the approval time needed in a branch does not require high levels of scalability. As a result, the minimum throughput and scalability requirements are selected as low.

One major situation about the notary services is considered as the increasing intensity of the volumes of documents. Hence, the minimum required level of storage is selected as high.

\subsection{Land Registry - Minimum Performance Requirements}

Land registry services can be considered as a subsegment of digital notary services. The only difference between the digital notary requirements is the volume of data that needs to be stored. It is considered lesser than the digital notary. Hence, the minimum storage requirement is set as moderate.

\subsection{Health Records - Minimum Performance Requirements}

It is required to have fast approvals of transactions of data in a network of hospital environment due to the criticality of individual health; therefore a Blockchain based immutable health records database minimally should need to have a moderate level of transaction rate of health recordings. The healths data is defined as laboratory test results, reports, prescriptions, diagnostic info and imaging data. It has to be also noted that due to the intensity of large amount of imaging data, the minimum required level of storage level is considered as high.

\subsection{Insurance - Minimum Performance Requirements}

In a global or multi-cliented realtime digital insurance scenario, daily and instant insurance contracts are being forecasted to be part of society's daily life. Anticipated participants will be mostly rental items such as automobiles. Due

www.astesj.com to the fact that; the number of registered validating nodes of an insurance company is considered as low enough. Hence, the scalability is selected as low. Whereas the speed of transactions should be moderately high enough in order to reduce the waiting durations during insurance processes. In addition to the transaction rate and scalability requirements, since the needed records data is text based, the minimum requirement for level of storage is determined as low. However, if there will be many items to be insured frequently then the requirement should be considered as moderate.

\subsection{E-Voting - Minimum Performance Requirements}

Electronic Voting, as one of the most suitable and politically sensitive application on the Blockchain and DLT domain, have high demanding transaction rate and scalability requirements. The setting environment in this application area is considered as fully mobile and distributed voting system. Hence, the energy efficiency requirement is selected as high. Given that; the necessity of having the voting/election as rapid as possible in a large scale voting event, the transaction rate requirement is also considered as high and due to the required mobile operation the scalability should also be considered as high.

\subsection{Energy Trade - Minimum Performance Requirements}

Blockchain-enabled Energy Trade sector is being regarded as a digital transformation technology in a Smart City context. In a practical sense, this concept is considered as a large scale IoT application in which the participants measure, share and certificate the energy production and consumption. With the inclusion of prosumers to the consumers and producers, the frequency of transactions of the energy certifications would be moderately high enough in a city/country wide environment. Hence, the minimum transaction rate is selected as moderate. Because of the prosumer and consumer-approval support for such an application, the minimum scalability requirement is considered as high. Because of the scenario of consumer-approvals and instant-billing and energy monitoring cases, the minimum energy efficiency should be selected as high. The data transactions are moderately high enough and the data size to be stored is essentially low. Hence, the minimum level of storage requirement should be kept as moderately high.

\subsection{DFS - Minimum Performance Requirements}

A globally effective Distributed File System should need to have immutable and zero-loss structure. It should be also accessible fast enough, the participating nodes should be fully decentralized and the number of nodes should be moderately enough. Energy efficiency is not considered as a requirement. Due to the variety of multiple data types such as text, audio, video and other possible large file types, the minimum required level of storage is considered as high.

. 


\subsection{AI/FL - Minimum Performance Requirements}

As a promising future application, federated learning and distributed neural networks model training will require high levels of data storage and fast execution times and multiple nodes.

\section{Applicability of Blockchain (PoW, PoS, PPoS) and DLTs on Modern Applications}

The Table 3 above summarizes Blockchain's (Note that Blockchain is considered as PoW, PoS, PPoS in this study) and new DLTs' suitability for the explained modern applications which are outlined throughout this study by following the information of minimum performance requirements per application given in Section 6 and Table 2. For better assessment the values in Table 1 is converted into the Low, Moderate, High performance scoring.

Table 3: Performance scoring levels of Blockchain (PoW, PoS, PPoS) and DLTs

\begin{tabular}{|l|l|l|l|l|}
\hline & $\begin{array}{l}\text { Throughput } \\
\text { (transactions } \\
\text { per second) }\end{array}$ & $\begin{array}{l}\text { Scalability } \\
\text { (number of } \\
\text { nodes) }\end{array}$ & Energy Efficiency & $\begin{array}{l}\text { Storage } \\
\text { Efficiency }\end{array}$ \\
\hline $\begin{array}{l}\text { Blockchain } \\
\text { - PoW }\end{array}$ & Low & Low & Low & Low \\
\hline $\begin{array}{l}\text { Blockchain } \\
\text { - PoS }\end{array}$ & Low & Low & Moderate & Low \\
\hline $\begin{array}{l}\text { Blockchain } \\
\text { - PPoS }\end{array}$ & Moderate & High & High & Low \\
\hline $\begin{array}{l}\text { DLT - } \\
\text { Hashgraph }\end{array}$ & Moderate & High & Moderate & Moderate \\
\hline $\begin{array}{l}\text { DLT - } \\
\text { Tangle }\end{array}$ & Low & High & High & Moderate \\
\hline $\begin{array}{l}\text { DLT - Holochain } \\
\text { How }\end{array}$ & High & High & High \\
\hline $\begin{array}{l}\text { DLT - } \\
\text { Tempo }\end{array}$ & High & High & High & High \\
\hline
\end{tabular}

Table 4 is constructed based on the scoring values of the performance criteria provided in Table 3 and minimum performance requirements provided in Table 2.

Table 4: Applicability of PoW, PoS, PPoS and DLTs on Modern Applications

\begin{tabular}{|l|l|l|l|l|l|l|l|}
\hline & PoW & PoS & PPoS & Hashgraph & Tangle & Holochain & Tempo \\
\hline $\begin{array}{l}\text { Crypto } \\
\text { Currency }\end{array}$ & $\checkmark$ & $\checkmark$ & $\checkmark$ & $\checkmark$ & $\checkmark$ & $\checkmark$ & $\checkmark$ \\
\hline DeFi & & & $\checkmark$ & $\checkmark$ & $\checkmark$ & $\checkmark$ & $\checkmark$ \\
\hline NFT & $\checkmark$ & $\checkmark$ & $\checkmark$ & $\checkmark$ & $\checkmark$ & $\checkmark$ & $\checkmark$ \\
\hline Digital ID & & & $\checkmark$ & $\checkmark$ & & $\checkmark$ & $\checkmark$ \\
\hline $\begin{array}{l}\text { Supply } \\
\text { Chain }\end{array}$ & & & $\checkmark$ & $\checkmark$ & & $\checkmark$ & $\checkmark$ \\
\hline $\begin{array}{l}\text { Digital } \\
\text { Notary }\end{array}$ & $\checkmark$ & $\checkmark$ & $\checkmark$ & $\checkmark$ & $\checkmark$ & $\checkmark$ & $\checkmark$ \\
\hline $\begin{array}{l}\text { Land } \\
\text { Registry }\end{array}$ & $\checkmark$ & $\checkmark$ & $\checkmark$ & $\checkmark$ & $\checkmark$ & $\checkmark$ & $\checkmark$ \\
\hline $\begin{array}{l}\text { Health } \\
\text { Records }\end{array}$ & & & $\checkmark$ & $\checkmark$ & & $\checkmark$ & $\checkmark$ \\
\hline $\begin{array}{l}\text { Insurance } \\
\text { Electronic } \\
\text { Voting }\end{array}$ & & & $\checkmark$ & $\checkmark$ & & $\checkmark$ & $\checkmark$ \\
\hline
\end{tabular}

\begin{tabular}{|l|l|l|l|l|l|l|l|}
\hline $\begin{array}{l}\text { Energy } \\
\text { Trade }\end{array}$ & & & $\checkmark$ & & & $\checkmark$ & $\checkmark$ \\
\hline DFS & & & & & & $\checkmark$ & $\checkmark$ \\
\hline AI/FL & & & $\checkmark$ & $\checkmark$ & & $\checkmark$ & $\checkmark$ \\
\hline
\end{tabular}

One major factor about the mapping of the consensus/DLT protocols is the distinction of consensus/protocol specific storage efficiency and application-specific minimally required storage levels. During the selection process of the suitable protocol, designer should be aware of that the needed amount in the peer's storage will be directly dependent on the selected protocol's storage efficiency and the desired application's required minimum level of storage. As an example, if the selected application requires high levels of storage and the selected consensus/protocol has low storage efficiency, then the resultant distributed system's peers will possibly require an enormous storage space. Hence, the storage related columns are not taken into consideration for the consensus/protocol selection process. The remaining Throughput, Scalability and Energy Efficiency criteria are taken into consideration for the selection of suitable consensus/protocol for the listed applications.

\section{Conclusions}

By deploying DLTs and Blockchain to suitable applications, parties can perform stable transactions for the first time in human history without relying on a centralized authority. Blockchain and Distributed Ledger Technologies have the power to modify most structures which are currently under central authority's control because of the concept of trust on peer transactions.

Blockchain infrastructures are inefficient in applications with high participation and transaction volume. As a result, new distributed ledger technologies have been created that primarily address the speed and scalability issues of Blockchain. Hashgraph, Tangle, Holochain and Tempo distributed ledger technologies are the recent alternatives to Blockchain. While these new distributed ledger technologies claim to solve the problems of Blockchain technology, their effectiveness has yet to be proven.

Participants and peers should should have the rights to conduct transactions without the use of intermediaries, and all Blockchain and Distributed Ledger Technologies should ensure decentralized management and participant data security. Under the hood of this prospect, a decentralized, flat and fully democratized structure with applications on Finance, Market Exchanges, Digital ID, Voting, Energy Trade, Distributed Storages, Insurance, Records Managements, Land Registry and AI will certainly constitute the future of humanity.

The completion of necessary country-wide regulations, as well as technical studies, will be a critical step toward the global adoption of blockchain and distributed ledger technologies, as well as related critical applications, in the near future.

\section{Conflict of Interest}

The authors declare no conflict of interest.

\section{Acknowledgment}

This study is fully supported by HAVELSAN Inc. R\&D, Technology and Product Management Directorate and HAVELSAN Blockchain and Distributed Systems Laboratory. 


\section{References}

[1] N. Chaudhry and M. M. Yousaf, "Consensus Algorithms in Blockchain: Comparative Analysis, Challenges and Opportunities," 2018 12th International Conference on Open Source Systems and Technologies (ICOSST), 54-63, 2018, doi: 10.1109/ICOSST.2018.8632190.

[2] S. Nakamoto, "Bitcoin: A Peer-to-Peer Electronic Cash System" [Online]. Available: https://bitcoin.org/bitcoin.pdf [Accessed: 05-08-2020].

[3] L. M. Bach, B. Mihaljevic and M. Zagar, "Comparative analysis of blockchain consensus algorithms," 2018 41st International Convention on Information and Communication Technology, Electronics and Microelectronics (MIPRO), 1545-1550, 2018, doi: 10.23919/MIPRO.2018.8400278.

[4] Cryptopedia, "Types of Blockchain: PoW, PoS, Private, and DLT" [Online], Available: https://www.gemini.com/cryptopedia/blockchain-types-powpos-private [Accessed: 01-Jul-2021]

[5] S.Haber, W.S.Stornetta, "How to time-stamp a digital document.", J. Cryptology 3, 99-111, 1991, doi:10.1007/BF00196791.

[6] A.Sunyaev, Distributed Ledger Technology. In: Internet Computing. Springer, Cham, 2020, doi:10.1007/978-3-030-34957-8_9.

[7] A. Panwar, V. Bhatnagar, "Distributed Ledger Technology (DLT): The Beginning of a Technological Revolution for Blockchain," 2nd International Conference on Data, Engineering and Applications (IDEA), 1-5, 2020, doi: 10.1109/IDEA49133.2020.9170699

[8] H. Alen, C. Tomislav \& Z. Ivana, Demystifying Distributed Ledger Technologies: Limits, Challenges and Potentials in the Energy Sector. IEEE Access, 7, 1-1. 2020. Doi: 10.1109/ACCESS.2020.3007935.

[9] Hedera Hashgraph, "Hedera: A Public Hashgraph Network \& Governing Council"

[10] F. M. Benčić and I. Podnar Žarko, "Distributed Ledger Technology: Blockchain Compared to Directed Acyclic Graph," 2018 IEEE 38th International Conference on Distributed Computing Systems (ICDCS), 1569-1570,2018, doi: 10.1109/ICDCS.2018.00171.

[11] N. Zivi et al., Directed Acyclic Graph as Tangle: an IoT Alternative to Blockchains. 1-3, 2019. 10.1109/TELFOR48224.2019.8971190.

[12] Holochain, "Holochain" [Online]. Available: https://github.com/Holochain/holochainproto/blob/whitepaper/holochain.pdf [Accessed: 06-08-2020].

[13] Ceptr, "Holochains for Distributed Data Integrity", [Online]. Available: http://ceptr.org/projects/holochain [Accessed:08-Jul-2020]

[14] Rutgers University, Logical clocks, [Online]. Available: https://www.cs.rutgers.edu/ pxk/417/notes/logical-clocks.html [Accessed:07-Jul-2021]

[15] Tempo, "Building Tempo" [Online]. Available: https://www.radixdlt.com/post/tempo-consensus-lessons-learned/ [Accessed: 05-08-2020].

[16] Cao, Bin \& Li, Yixin \& Zhang, Lei \& Zhang, Long \& Mumtaz, Shahid \& Zhou, Zhenyu \& Peng, Mugen. (2019). When Internet of Things Meets Blockchain: Challenges in Distributed Consensus. IEEE Network. PP. 1. 10.1109/MNET.2019.1900002.

[17] K. Enis Data Storage in the Decentralized World: Blockchain and Derivatives, 2020. Doi: 10.26650/B/ET06.2020.011.03.

[18] Holochain, "What is the TPS (Transactions Per Second) on Holochain? Learning Library / FAQs - Holochain Forum" [Online]. Available: https://forum.holochain.org/t/what-is-the-tps-transactions-per-second-onholochain/191 [Accessed: 08-Jul-2021]

[19] Radix DLT Tempo, "10 years of Bitcoin history, replayed in under 30 minutes | The Radix Blog | Radix DLT" [Online], Available: https://www.radixdlt.com/post/replaying-bitcoin [Accessed: 01-Jul-2021]

[20] Holochain, "The Holochain Guidebook FAQ",

[21] Hedera Hashgraph, "Hedera Hashgraph", [Online]. Available: https://hedera.com/ [Accessed: 05-Jul-2021]

[22] Lepore, Cristian \& Ceria, Michela \& Visconti, Andrea \& Rao, Udai Pratap \& Shah, Kaushal \& Zanolini, Luca. (2020). A Survey on Blockchain Consensus with a Performance Comparison of PoW, PoS and Pure PoS. Mathematics. 8. 1782. 10.3390/math8101782.

[23] Sedlmeir, J., Buhl, H.U., Fridgen, G. et al. The Energy Consumption of Blockchain Technology: Beyond Myth. Bus Inf Syst Eng 62, 599-608 (2020). https://doi.org/10.1007/s12599-020-00656-x

[24] Sustainable Blockchain: Estimating the Carbon Footprint of Algorand's Pure Proof-of-Stake | Algorand, [Online]. Available: https://www.algorand.com/resources/blog/sustainable-blockchaincalculating-the-carbon-footprint . [Accessed:07-Jul-2021]
[25] IOTA, “Tangle Energy Benchmarks for IOTA", [Online]. Available: https://blog.iota.org/internal-energy-benchmarks-for-iota/ [Accessed: 06Jul-2021]

[26] Leafscore, "Sustainable Crypto 2021”, [Online]. Available: https://www.leafscore.com/blog/the-9-most-sustainable-cryptocurrenciesfor-2021/ [Accessed:07-Jul-2021]

[27] Holochain, "Holoport Electricity Usage", [Online]. Available: https://help.holo.host/support/solutions/articles/36000035595-will-holouse-lots-of-electricity-how-much-electricity-will-my-holoport-use[Accessed: 07-Jul-2021]

[28] A. Hany \& W. Gary. Intersections between IoT and distributed ledger, 2019. Doi: 10.1016/bs.adcom.2018.12.001.

[29] Hashemi Joo, M., Nishikawa, Y. and Dandapani, K., "Cryptocurrency, a successful application of blockchain technology", Managerial Finance, Vol. 46 No. 6, 715-733,2020, doi:10.1108/MF-09-2018-0451

[30] Decrypt, "What Are Privacy Coins? Monero, Zcash, and Dash Explained Decrypt" [Online]. Available: https://decrypt.co/resources/what-areprivacy-coins-monero-zcash-and-dash-explained [Accessed: 30-Jun-2021]

[31] Ethereum, "Decentralized finance (DeFi)" [Online]. Available: https://ethereum.org/en/defi/ [Accessed: 30-Jun-2021]

[32] Investopedia, "DeFi (Decentralized Finance) Definition and Why it Matters" [Online]. Available: https://www.investopedia.com/decentralized-financedefi-5113835 [Accessed: 29-Jun-2021]

[33] Cryptopedia, "Non-Fungible Tokens (NFTs): Crypto Collectibles | Gemini" [Online]. Available: https://www.gemini.com/cryptopedia/nft-non-fungibletoken-crypto-collectibles [Accessed: 29-Jun-2021]

[34] M. Takemiya and B. Vanieiev, "Sora Identity: Secure, Digital Identity on the Blockchain," 2018 IEEE 42nd Annual Computer Software and Applications Conference (COMPSAC), 582-587,2018, doi 10.1109/COMPSAC.2018.10299.

[35] Consensys, "Zug Digital ID: Blockchain Case Study for Government Issued Identity" [Online]. Available: https://consensys.net/blockchain-usecases/government-and-the- public-sector/zug [Accessed: 17-08-2020].

[36] N.Hackius, M.Petersen "Blockchain in Logistics and Supply Chain : Trick or Treat?”, Epubli, 23, 2017, doi:10.15480/882.1444

[37] Gao, Ying \& Pan, Qiaofeng \& Liu, Yangliang \& Lin, Hongliang \& Chen, Yijian \& Wen, Quansi. (2021). The Notarial Office in E-government:A Blockchain-Based Solution. IEEE Access. PP. 1-1. 10.1109/ACCESS.2021.3066184.

[38] Barbieri, Maurice, and Dominik Gassen. "Blockchain-can this new technology really revolutionize the land registry system." Responsible Land Governance: Towards an Evidence Based Approach: Proceedings of the Annual World Bank Conference on Land and Poverty. 2017

[39] Agbo, C.C.; Mahmoud, Q.H.; Eklund, J.M. Blockchain Technology in Healthcare: A Systematic Review. Healthcare 2019, 7, 56, doi:10.3390/healthcare7020056

[40] Anokye Acheampong AMPONSAH, Adebayo Felix ADEKOYA and Benjamin Asubam WEYORI, "Blockchain in Insurance: Exploratory Analysis of Prospects and Threats" International Journal of Advanced Computer Science and Applications(IJACSA), 12(1), 2021. http://dx.doi.org/10.14569/IJACSA.2021.0120153

[41] N. Kshetri, J. Voas, "Blockchain-Enabled E-Voting," in IEEE Software, 35(4), 95-99, 2018, doi: 10.1109/MS.2018.2801546.

[42] R. Hanifatunnisa, B. Rahardjo, "Blockchain based e-voting recording system design," 2017 11th International Conference on Telecommunication Systems Services and Applications (TSSA), 2017, 1-6, doi: 10.1109/TSSA.2017.8272896.

[43] Andoni, V. Robu, D.Flynn, S. Abram, D. Geach, D.Jenkins, P. McCallum, A.Peacock,"Blockchain technology in the energy sector: A systematic review of challenges and opportunities",Renewable and Sustainable Energy Reviews, 100,143-174,2019,doi:10.1016/j.rser.2018.10.014.

[44] K. Mannaro, A. Pinna, M. Marchesi, "Crypto-trading: Blockchain-oriented energy market," 2017 AEIT International Annual Conference, 1-5,2017, doi: 10.23919/AEIT.2017.8240547.

[45] H. Huawei et al., When Blockchain Meets Distributed File Systems: An Overview, Challenges, and Open Issues. IEEE Access, 7, 2020 10.1109/ACCESS.2020.2979881.

[46] M. Chuan et al., When Federated Learning Meets Blockchain: A New Distributed Learning Paradigm, IEEE Access, 7, 1189, 2020. Doi: 10.1109/ACCESS.2020.2971189. 